\title{
DOI: https://doi.org/10.24297/jap.v18i.8811
}

\section{Magnetism: Entanglement, Magnetism as Discrete, Quantum Computing Solutions?}

\author{
Bhekuzulu Khumalo \\ 305 - 19 Northern Heights Dr. Richmond Hill, Ontario, Canada \\ bhekuzulu.khumalo@gmail.com
}

\begin{abstract}
Understanding that everything is in a relationship led us to ask what then is the relationship that caused particles to be. Our investigation led us to understand that all particles are made up of the same stuff we called konke. Something that has experimental backing. Understanding that this konke must get into relationships so that we get particles, we have a chance to look at particles from what they are made off understanding; it is how much of konke that gives a particle mass and its categorization. What is the nature of magnetism from the viewpoint that all things are made of the same stuff, konke? Two simple experiments are requiring a vacuum that will help solve the nature of magnetism, that should end up as a discrete phenomenon, we can measure its speed, mass, polarization like other phenomena. Rely on experimentation as much as possible. Implications in quantum computing though possible variations in the experiments.
\end{abstract}

\section{Keywords: discrete magnetism, entanglement, magnetic fields, monopole, quantum computing}

\section{Introduction}

In the paper "Konke, Gravity, Particle Relationships" it was explained that all particles are made of the same stuff and this is proven in particle colliders and the creation of neutrinos in the stars.

The paper explained how particles are differentiated by how much mass they have. We know if a particle has a certain mass it can for example be considered an electron, or a quark, or a photon, or a photon. Particles can further be classified by relationships of the material that make them up. Two particles can have the same mass, but internal relationships differentiate them. For example, a radio photon has the same mass as a gamma photon, but their internal structures are different giving them different properties.

It was shown in the above-mentioned paper that this stuff that every particle is made of, konke, needs an agent to have a relationship and thus attain the state of what we have termed mass. This second variable, $V_{2}$ was shown that it must be gravity. This massless matter can not break the rules of relationships, namely the rule that everything is in a relationship and all relationships need energy to maintain thus leading to a loss of freedom. For konke to get into a relationship there must be a loss of freedom hence the need for Variable $2, V_{2} . V_{2}$ represents the energy needed for konke to have a state of what we consider mass, so that konke is structured as mass.

Given that we know that there are basic ${ }^{2}$ characteristics of information that affects all information, and everything is information because it will all inform you about what it is by observation. These basic characteristics of information are all information is:

- Discrete

- Independent

- Can be categorized

- Consistent (why it is important to understand logic)

- Can be used to compute

Given that all information is discrete it means no information can be continuous. A photon is a discrete particle, an electron is a discrete particle. The stuff that makes up all particles konke must itself be discrete as we can see particles with different amounts of it, these amounts determined by how much mass a particle has. 
Magnetism is information, we can see its actions, we know this or that is magnified. We have ${ }^{3}$ natural magnets as well artificial magnets. Natural magnets occur like lodestone, magnetite, and pyrrhotite. Their magnetic fields are weak, but those magnetics fields exist. Other natural magnets are solar bodies like planets and stars. This planet some call earth, that a name, in other languages it is called place of trauma, this planet like others has a magnetic field, a north and a south pole. The magnetism in stars is thought to be generated by ${ }^{4}$ convection within the stars. Just as it is thought magnetic field of earth is convection within the core, not all planets have a magnetic field. This is not important, what is important is stars and some planets have magnetic fields.

Artificial magnetic fields include bar magnets. We use iron because the electrons in iron most of the electrons spin in the same direction. But in most materials' half the electrons flow in opposite directions, thus they are not suitable as good artificial magnet material.

There can be said to be three classes of magnets,

- Natural like lodestone

- Stars and some planets

- Unnatural magnets like a horseshoe magnet

Natural magnets like lodestone are there, they are magnetic there is no convention or electricity applied, they exist. Planets and stars are magnetic dues to convention inside their bodies. Unnatural magnets are created by humans, running an electric current through them. Magnets can also be created by using a grinder to cut down iron. For example in construction sites when rebar is cut it becomes hot and magnetic. It retains this magnetism even after it cools. This is shown in photo 1 below. The rebar was just cut using a gas powered grinder.

What is this magnetic given it must be discrete like everything else in existence? We must answer this question in such a way that an experiment can be proposed that makes sense, and is simple enough. What a magnet is must satisfy all conditions of a magnet at all times, be it a natural magnetic field or an unnatural magnetic field as that around bar magnets.

\section{Aims}

The aim of this paper is to look at a magnet as a discrete phenomenon and as to how we understood everything is made from konke from experiments in colliders and the behaviour inside stars that creates neutrinos. The aim of this paper is to show that magnetic fields too are from this konke and show with experimental proof.

This paper therefore is looking $t$ the magnet from the reality that everything is in a relations and all relationships result in a loss of freedom due to the energy needed to maintain them, and all relationships are random.

The idea is to start accepting that all information is discrete, and this donson, the magnetic particle must exist. Experiments take out any personal feelings towards something. The idea should be simple enough that a 14 year old can understand.

\section{Significance}

The significance of this paper is that whilst looking at what a magnet is, we will see that this konke is everywhere. Just as one can create a coolider anywhere and bump particles together and get to konke, one can create a magnet anywhere given the right conditions. Magnetism shows us that we interact with konke at will. An experiment will prove this. But we must first go into the background of why there can be an experiment, technically not exceedingly difficult.

\subsection{Recap, the inside workings of a particle}

In the paper, ${ }^{1}$ Konke, Gravity, Particle relationships it was shown that konke needs to get into a relationship for us to get the state that we consider mass. Figure 1 is from that paper. 


\section{Figure 1: Konke in Relationships}

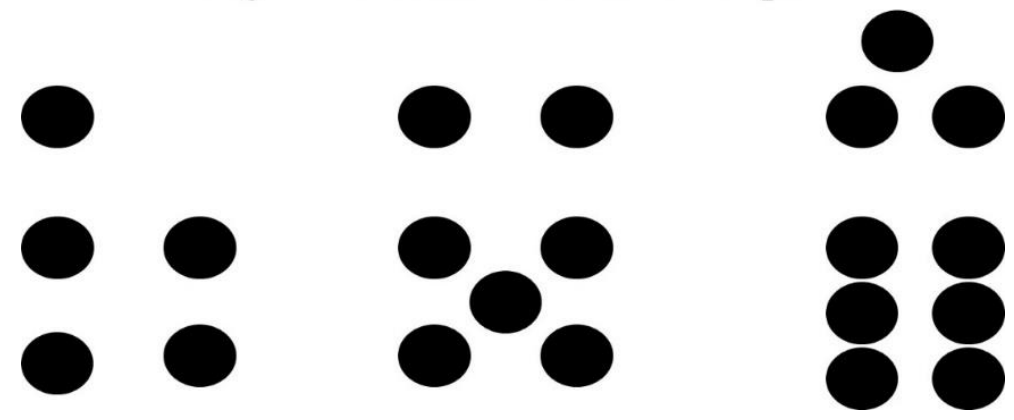

Figure 1 shows an illustration of possible relations of konke that lead to mass and the size of the mass. These representations of konke relationships are made simple in the form of patterns in a dice. The problem with figure 1 is if this was true, when one smashes particles in a collider, they do not magically come back as a particle, rather they are unstable. This means that another agent is needed so that konke can get into a relationship that will result in a loss of freedom. Figure 2 also comes from the above paper, it

Figure 2: Konke in Relationships $V_{2}$
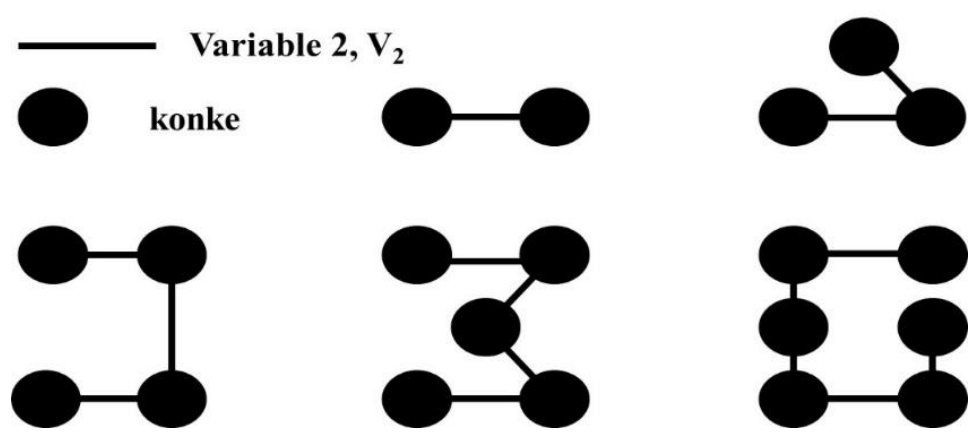

Figure 2 shows another variable added that was called variable 2, or $V_{2}$. This is the agent under certain conditions will allow konke to get into a relationship. As all relationships need energy to be maintained, $V_{2}$ is this energy. Figure 2 is a more realistic depiction of the inside workings of a discrete piece of information that has attained mass. We can see that each discrete piece of information that has attained mass has different amounts of konke, thus different amounts of mass and different characteristics.

Though particles of the same mass are classified as similar particles, for example all photons have the same amount of mass, but all different differently, some are blue, some are red, some are yellow, others are radio waves, others are gamma rays. This phenomenon is illustrated in figure 3.

One gets the general understanding same mass similar type particle. One can become specific however and mention phenomenon like electrons and positrons having similar mass but anti particles of each other. In the paper $\#^{2}$ The Algorithm of Information and the Origin of Basic Particles, It was mentioned, "It is here we understand what happens when an antiparticle meets a particle, everything just goes to this everything that makes up all particles. Just that with the antiparticle it is immediate whilst with smashing neutrons together we first get unstable particles then it's gone to this everything" Why does the anti-particle have the same mass and capability of annihilating each other. The anti particle is essentially the same particle that has the configuration of konke set in such a way that if the 2 particles meet, they become unstable and return to what created both particles. Konke being the everything that the particles return to.

One could also argue a photon and a graviton have the same mass and they are not similar. That is a bold assertion. What if Variable 2 above is indeed gravity? What is the bonding energy that gives konke ability to have mass? There are a lot of questions that still must be answered around gravity. To then say a graviton has same mass as a photon is wrong when gravity could very well make up every particle. Let us get to the experiments at the end of this paper first. 


\section{Figure 3: Variations in Same Particle}

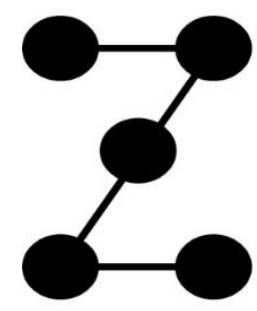

$5 \mathbf{a}$

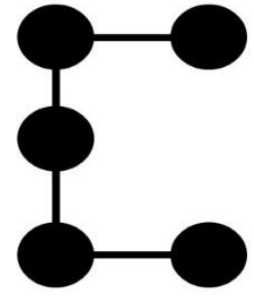

$5 \mathbf{b}$

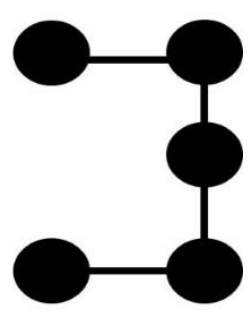

5c

Figure 3 shows information with the same amount of mass, but because of the different configurations of konke the particles do not have exactly the same properties, just equal amounts of mass. It is from figure 3 that we shall build a reality confirming to the behaviour of a magnet, at the end, an experiment shall be provided.

\subsection{The discrete magnet/ Donson}

For a magnetic force to be considered discrete it is a particle, this particle has been named a donson.

\section{Figure 4: illustration of a Donson}

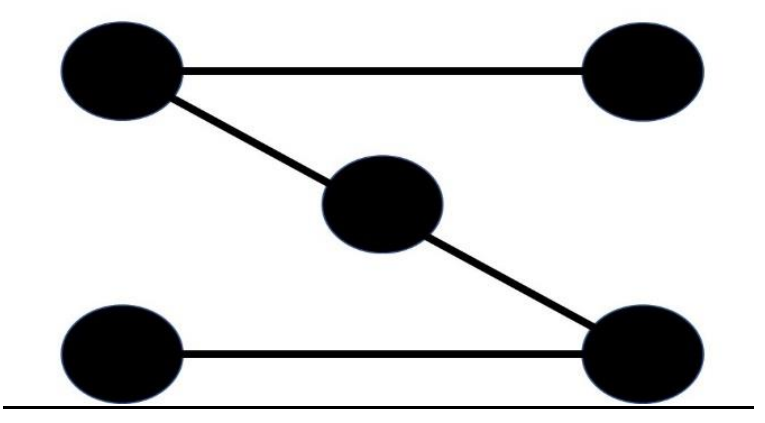

Figure 4 is an illustration of a donson, there is konke and $\mathrm{V}_{2} \underline{V}_{2}$ can be considered gravity and it was explained in the paper "Konke, Gravity, Particle Relationships".

\subsection{Magnetic Fields}

Having an illustration in our mind for a donson, figure 4, just so that we can conceptualize the coming argument, we need to look at a magnet. A magnet has a north and south pole and has a field running through it as illustrated in figure 5.

Figure 5: A Magnetic Field

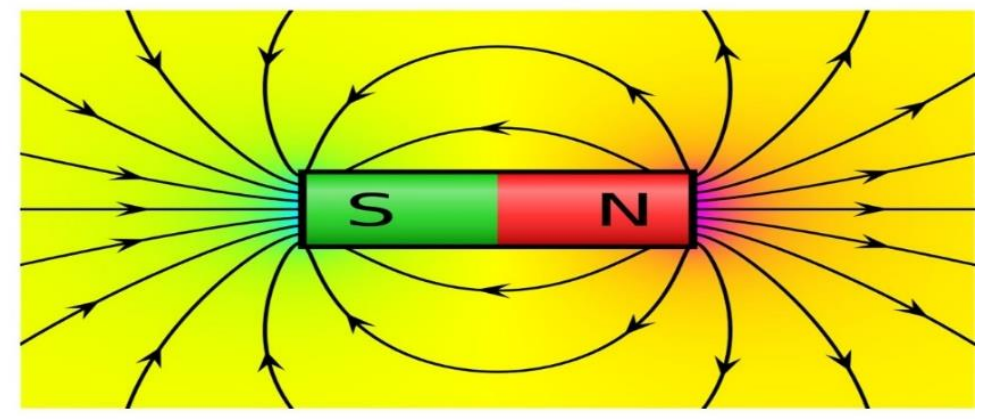

Source: Geek3, wikicommons 
Figure 5 is a typical magnetic field, half the field is North and half the field is South and is strongest at the poles. But everything is discrete, everything can't be discrete except for 2 or 3 phenomena given the multitude of variety that is all discrete. Take 4 locations in the magnetic field, these beng 1,2,3, and 4 as depicted in figure 6.

Figure 6: A Magnetic Field/ Donson

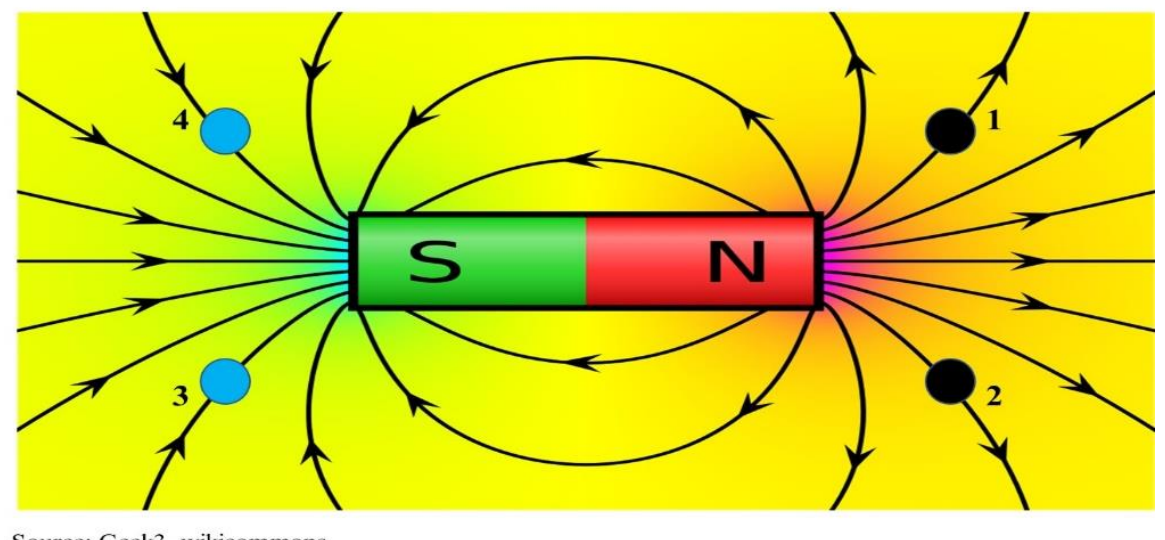

Source: Geek3, wikicommons

As we can see from figure 7 there are 4 discrete locations. Two correspond to the north pole influence these being 1 and 2. Two correspond to the south pole these being 4 and 3 .

\subsection{Natural Entanglement}

\subsubsection{Entanglement}

Entanglement is one of the most important basics of quantum mechanics. We know a particle can have two states, say polarization. If two particles are entangled, if we measure one particle and get a result we know the other particle is the other state. What we know about entanglement thus far can be summarized as " In quantum entanglement, the physical measurements (such as position, momentum, spin, and polarization) of two entangled particles are highly correlated, even though they could be light years apart from each other."

\subsubsection{Magnetism and Natural Entanglement}

Let us take those discrete locations 1,2,3, and 4 in figure 6 as donsons, discrete magnetic particles and we get figure 7 . In this figure we have four particles $1,1^{\prime}, 2$, and $2^{\prime}$.

Figure 7: A Magnetic Field/ Donson 2

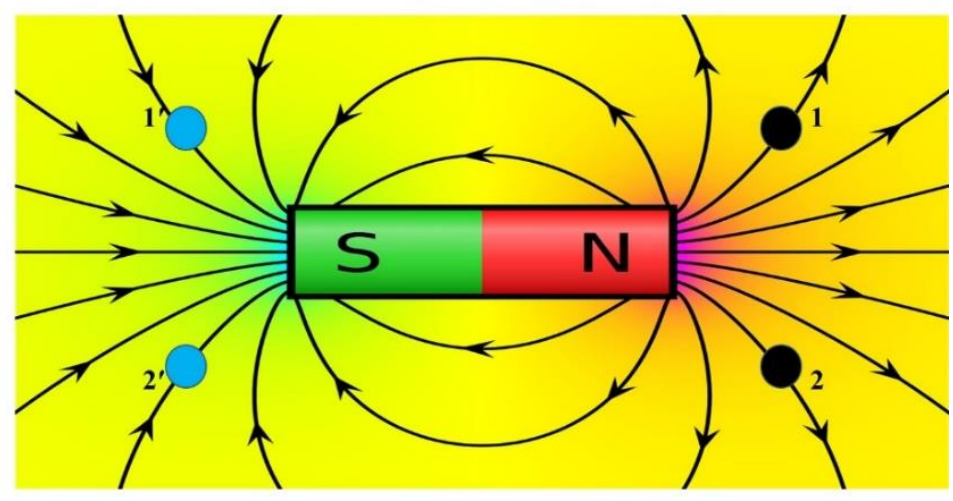

Source: Geek3, wikicommons

The naming of the particles is done on purpose. 1 represents a magnetic particle, the donson that is north polarized and $1^{\prime}$ that is south polarized. 2 is north polarized and 2 ' is south polarized. The magnetic field is like that. There are the same number of donsons, half north have south. Each north has a corresponding south. That is what can be considered natural entanglement, except in this instance we know the polarization of the particle 
and we will know the polarization of the corresponding particle. By knowing the position of say 1 in the magnetic field we will know the position and polarity of $1^{\prime}$.

Natural entanglement represents entanglement that is predictable, that is what theoretically a donson implies. Figure 7 is just a for us what is going on, there is entanglement. However, figure 8 will probably be a more accurate description.

Figure 8: Realistic Position of Donson

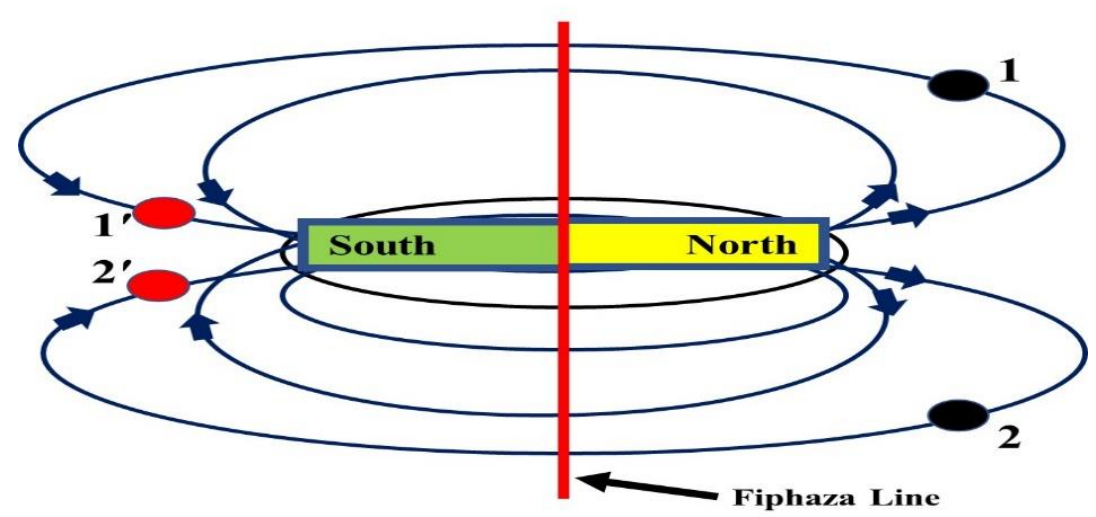

Imagine figure 8 having the same quality and principles as figures $5-7$, difficult to replicate using power point but hopefully we will understand something especially important about "natural" entanglement. The implications confirm so much.

In figure 8, we have donson 1 and 2 and they are polarized to the north. 1' and 2' are polarized to the south. Given that there must always be half north and half south of the total quantity of donson, don't worry how the magnetic field comes to be, it is there, be it artificial like a bar magnet, be it natural like lodestone or celestial like a planet or a star.

One could argue that they can not understand how 1,2,1', and $2^{\prime}$ are particles, what is their mass? what is the charge, well they are either north or south. what is the velocity, the velocity depends on what entanglement is. This is a theory being put forward, reading further one can read the experiments, we will get the mass, we will know the polarity because of position, north or south, and the velocity will answer many questions around entanglement but not all. You need to do an experiment first to determine the reality. Why calculate the reality when one can measure. One must read further.

The number of donsons obviously determines the strength of the magnetic force so there cannot be infinite number of donsons around making up a magnetic force.

\section{Figure 9: Magnetized Rebar after being cut by a gas powered cutter}

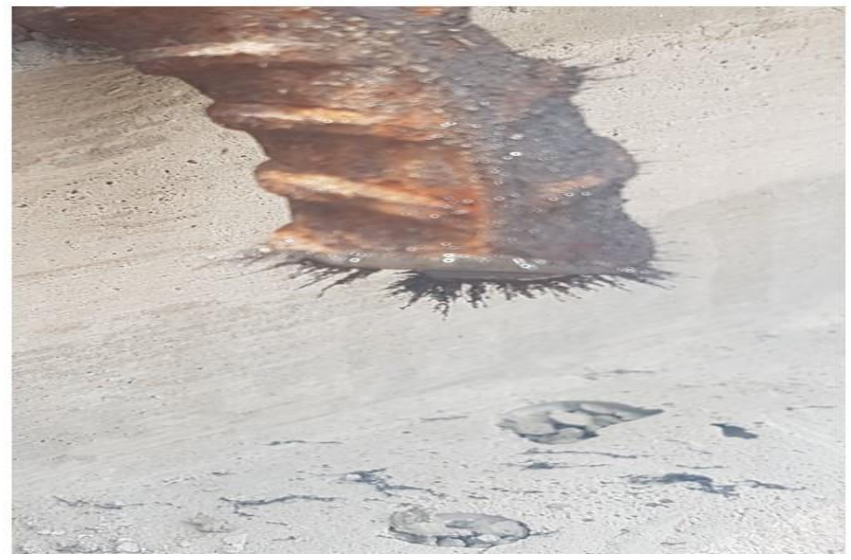


The reason why figure 8 would be a more accurate representation than figure 7 is that the particles are entangled, for every particle that is north there is another one that is south. If a particle that is north becomes south there must be a corresponding particle that turns from south to north polarization to maintain the balance. Thus 1 must be equidistance from the fiphaza line with $1^{\prime}$.

The fiphaza line is the difference between north and south. For example, in figure 8, on the right of the fiphaza line we have north and on the left we have south. 2 must have the same amount of travel time to get to the fiphaza line as 2'. They must cross the fiphaza line at the same time such that 2 switches to south at the same instance as $2^{\prime}$ turns to north polarization.

Polarization is then just about the position of the relationships inside the particle just like green and yellow are just about positions and locations of material even though they all have the same amount of mass.

Figure 10 has the same principles as figures 3 and 5 . It is just so that we understand the internal workings of what must be happening as a donson changes from north polarization to south polarization. This happens at a zone we have termed the fiphaza line.

Figure 10: Happenings at the Fiphaza Line
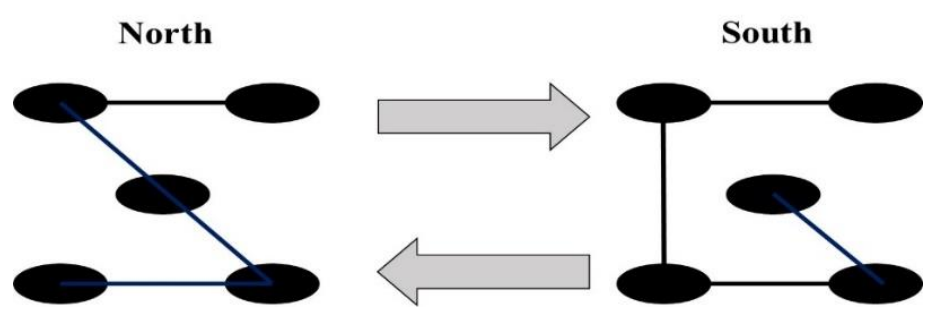

At that line to keep the balance, for any particle changing polarization, there must be another particle to change polarization and take its place.

The fiphaza line is theoretically the shortest distance that an action can take place in existence.

\subsection{What can be Tested}

What has been written above is theory. No matter how much logic and sense it makes it remains a theory. What is real is what we can measure. How can we test the nature of magnetism, what is it? We can only answer by what we can test.

\subsection{Can we see Relationships in Konke in Magnetism?}

Konke is what we have termed, what we have named the thing that every single piece of mass consists of in existence, all particles are made up of the same stuff. Can one safetly form a hypothesis that magnetism, the magnet is stuff that is very close to konke. To satisfy this hypothesis it will have to be proven that a magnet is its own thing, a unidue existence separate from other forces. To do this we can not use a natural magnet because the field is on, we need to use an artificial magnet in a vacuum. If off the material attracts nothing very well, but if in a vacuum an artificial magnet attracts something, that means something else exists separate from the source, yes it is that simple. An artificial magnet if off in a vacuum attracts nothing but once on, it means there is something unique, it can only be a unique force, not electro magnetism, magnet, because if an artificial magnet can attract in a vacuum, it means a lodestone can as well. It means the current allows us to interfere with this thing that everything is made from and we get a magnetic field.

Because we know if we create a magnetic field there will be attraction in a vacuum, if we put a natural magnte in there, that attraction means that space is filled directly from interference with konke, what every particle is made of, thus nullifying the vacuum as relationships have been caused in konke that there is a magnet.

This is experiment is illustrated in figure 11, things need not be as complicated as people wish them to be. Figure 11 shows a proposed experiment. 


\section{Figure 11: Creating Magnetic Field in a Vacuum}

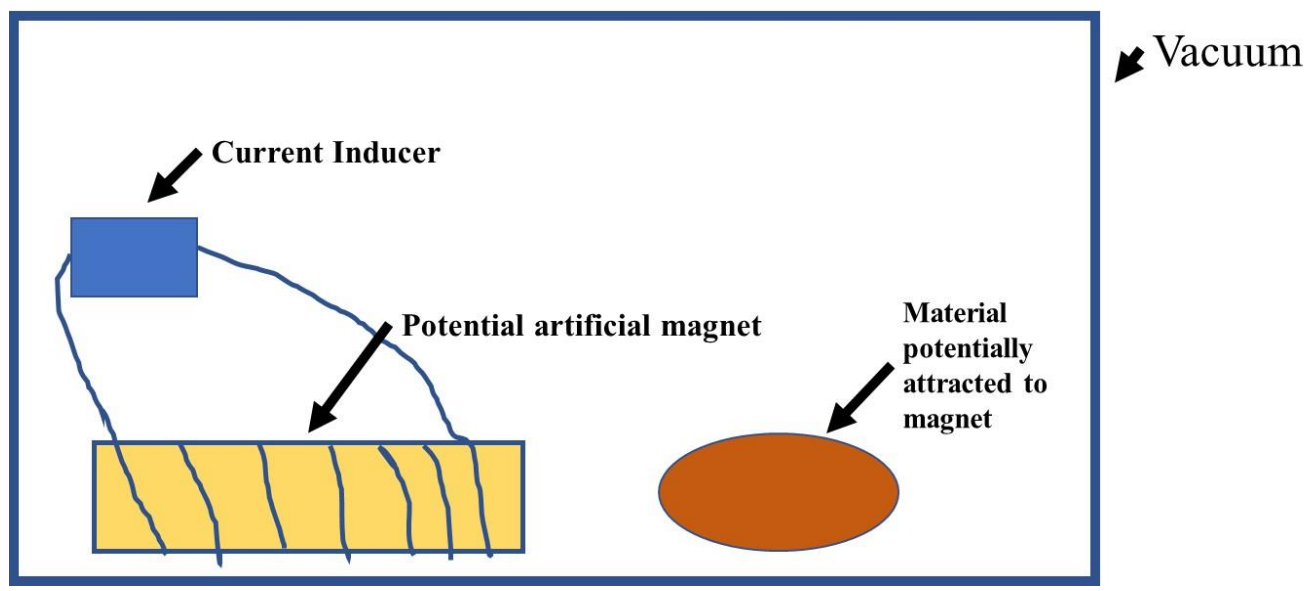

Figure 11 show a proposed experiment. We have a vacuum, there is no obstruction from any source except gravity. We have material that can be artificially magnetized, a current inducer to magnetize the material that can be artificially magnetized. There is also a material that is potentially attracted to a magnet.

Switching on the current inducer, the material that is a potential magnet should become magnetized. If it attracts the material then we have an independent, discrete force that is a result of inducing an electric current through say an iron bar. This independent force, can only be the result of that when an electrical current is induced through an iron bar, a relationship is created that affects the konke, the thing that all particles are made from and we get magnetic particles. This is humans directly interfering with the stuff particles return to in the particle collider, in front of us all the time, since artificial magnets where created.

The experiment in figure 11 represents a step-in knowledge knowing that we can interfere with konke and create what we can measure. This knowledge we can only gain because we can experimentally create magnetic fields.

Figure 11 means that we can interfere with konke and create a field a donson. Maybe not all basic particles, but we can create one. Surely the signal of man's advancement was inducing an artificial magnetic field.

Figure 12 involves further ambition. What if we put a scale beneath the vacuum what will we see?

Figure 12: Does a Magnet Have Mass

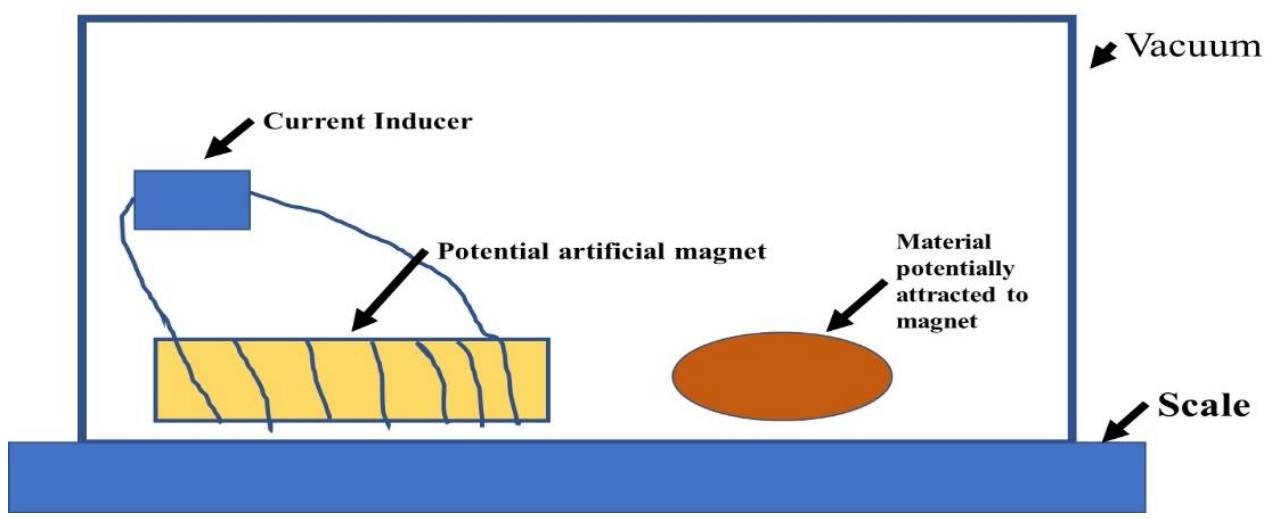

We shall not guess here but look at the results. That is what experiments are for, to gain knowledge and verify. We can not enter this one with preconceived ideas. There are valid arguments for either way the experiment goes. There are three possibilities, gain of mass, loss of mass, no change in mass, science is an adventure, that is why we do experiments.

Let us suck out as much information from the experiments, something thanks to my referees. Besides confirming the existence of this new phenomenon in a vacuum, we can measure the speed of this phenomenon if we so 
desire. This is merely measuring how long it takes the magnetic force to affect the potentially magnetic material. One can guess near the speed of light. This will lead to further debates around the nature of entanglement. Because of the politics around entanglement it's speed, one can think of the implications themselves.

Are we sure the phenomenon we are dealing with is konke. Could not there be other interference. What other phenomenon can be occurring? Could it have something to do with neutrinos as they pass through most things known? But neutrinos don't react with things, but we can find from this phenomenon how its introduction affects the behaviour of neutrinos.

The experiment can be done in space to see the effect of gravity on the phenomenon.

With sophistication we can test particle annihilation and entanglement. When one particle is annihilated what happens to it's opposite. How quickly are the annihilated particles replaced? They must be replaced because the magnetic field must keep its strength. Because we know where the position of the opposite particle in terms of polarization ought to be, what happens when we interfere with one sector of field, is there a corresponding effect without any interference to the opposite sector. We are talking possible high technology implications, controlled sustainable entanglement. Something a quantum computer desires, meaning magnetism will not be a by product of transistors but what does the computing. Not forgetting that everything is information and one of the properties of all information is that it can be used to compute, from cows, beads, to electrons, to donsons, the given name of the magnetic particle.

\section{Conclusions}

We are looking at relationships from as deep as experimentally possible. We know everything is made up of the same stuff, every particle, we have called this same stuff konke. This stuff must get into relationships so that we get particles, these relationships will require energy to maintain so that we can have the structure of particles.

What differentiates particles their mass and organization of konke if they have the same mass. The paper has theorized about the magnet, but theory is theory, what can we prove, what can we test, what can we experiment on?

Two experiments have been offered. The first experiment proves that with artificial magnetism we are tinkering with konke and creating relationships that give us a magnetic field. It is an artificial magnetic field. But we know natural magnetic fields like the one around the earth help us to survive on this planet protecting us from harmful cosmic rays. One would expect artificial magnets to have the same properties.

But in time, it is possible that we find differences between artificial magnets and natural magnets. Just as there is variety amongst photons and neutrinos. But magnetic particles can only exist in pairs.

Experiments are the way. Alexander Ivanchin in his paper concluded that "6rom the article presented it follows that there is close interdependence between the electric and gravitational fields. According to [9] , the magnetic field is the manifestation of the properties of the electric field. Thus, all the three fields, the electric, magnetic and the gravitational ones, are different manifestations of the electric field. The gravitational field is caused by the properties of the vacuum that responds differently to the positive and negative charges." Obviously, this paper does not agree with this, we look at it from konke, how did konke get into relationships. But it agrees there is a relationship between gravity and magnetism. Thus, why the need for the second experiment, having a relationship with gravity implies mass.

Yin Zhu in his paper says, "The equations show that the variation of the gravitational field could be measured. And, we found that, the energy density of the magnetic field is close to that of the gravitational one." Thus, again for the second experiment it would not be far fetched to expect increases in mass.

Both Zhu and Ivanchin, and the science in community in large needs to accept the reality that all particles are made of the same stuff and we have called this stuff konke. Start from what is the agent that causes konke to get into a relationship and we have mass as we know it. It does not start with the particle.

\section{Acknowledgements}


The author accepts that everything is information and had once believed this reality came from Susskind and Leggett, no harm the term comes from ${ }^{8} \mathrm{~J}$ ohn Archibald Wheeler. If it was not for him we would not know that everything is in a relationship and all relationships need energy to maintain thus resulting in a loss of freedom and structure, it works that way. What is it that we know? We know information and it is all made up of the same stuff we have categorized as konke and it is all in a relationship.

\section{References}

${ }^{5}$ Duan, D. (2019) Seeing is Believing: The First-ever Image of Quantum Entanglement https://www.labroots.com/trending/chemistry-and-physics/15218/seeing-believing-first-ever-imagequantum-entanglement

${ }^{6}$ Ivanchin, A. (2017) "Electric, Magnetic, Gravitational Field of the Earth https://www.scirp.org/journal/paperinformation.aspx?paperid=81107\#ref9 DOI: 10.4236/gep.2017.512005

${ }^{2}$ Khumalo, B. (2019) "The Algorithm of Information and the Origin of Basic Particles" https://rajpub.com/index.php/jap/article/view/8485 DOI: https://doi.org/10.24297/jap.v16i1.8485

${ }^{1}$ Khumalo,B. (2020) "Konke, Gravity Particle Relationships" https://rajpub.com/index.php/jap/article/view/8794 DOI: https://doi.org/10.24297/jap.v18i.8794

${ }^{4}$ Mathewson, S. (2016) Sun-Like Stars Generate Magnetic Field in Unexpected Way https://www.space.com/33607-sun-like-stars-magnetic-field-generation.html

${ }^{8}$ Paerry, P. (2020) There is no dark matter. Instead Information has mass, physicist says https://bigthink.com/surprising-science/dark-matter-theory?rebelltitem=6\#rebelltitem6

${ }^{3}$ Teachoo. (2020) What is the difference between Natural and Artificial Magnets? https://www.teachoo.com/10750/3117/What-is-the-difference-between-Natural-and-Artificial-Magnets/category/Extra-Questions/

7Zhu, Y. (2018) "Gravitational-magnetic-electrical field interaction" https://www.sciencedirect.com/science/article/pii/S2211379718314128

https://doi.org/10.1016/j.rinp.2018.07.029 